\title{
Rapid Preparation of Mesoporous Methylsilsesquioxane Aerogels by Microwave Heating Technology
}

\author{
Xingzhong Guo ${ }^{1,2, *\left(\mathbb{D}, \text { Zixiao Li }^{1} \text {, Wei Lei }\right.}{ }^{2}$, Ronghua Ding ${ }^{2}$, Yun Zhang ${ }^{2}$ and Hui Yang ${ }^{1}$ \\ 1 State Key Laboratory of Silicon Materials, School of Materials Science and Engineering, Zhejiang University, \\ Hangzhou 310027, China; 21826012@zju.edu.cn (Z.L.); yanghui@zju.edu.cn (H.Y.) \\ 2 Pan Asia Microvent Tech (Jiangsu) Coporation \& Zhejiang University Micro-Nano-Porous Materials Joint \\ Research Development Center, Changzhou 213100, China; leiwei@microvent.com.cn (W.L.); \\ dingronghua@microvent.com.cn (R.D.); zhangyun@microvent.com.cn (Y.Z.) \\ * Correspondence: msewj01@zju.edu.cn; Tel./Fax: +86-571-87953313
}

Citation: Guo, X.; Li, Z.; Lei, W.; Ding, R.; Zhang, Y.; Yang, H. Rapid Preparation of Mesoporous Methylsilsesquioxane Aerogels by Microwave Heating Technology. Molecules 2021, 26, 1960.

https://doi.org/10.3390/molecules 26071960

Academic Editor: Mihkel Koel

Received: 4 March 2021

Accepted: 29 March 2021

Published: 31 March 2021

Publisher's Note: MDPI stays neutral with regard to jurisdictional claims in published maps and institutional affiliations.

Copyright: (c) 2021 by the authors. Licensee MDPI, Basel, Switzerland. This article is an open access article distributed under the terms and conditions of the Creative Commons Attribution (CC BY) license (https:// creativecommons.org/licenses/by/ $4.0 /)$.

\begin{abstract}
Microwave heating technology is known as an alternative to traditional gas and electric heating sources. In this work, mesoporous methylsilsesquioxane (MSQ) aerogels were prepared via a sol-gel process accompanied by microwave heating technology, and microwave heating was used in the gelation of sol and the drying of wet gels, respectively. The effects of hexadecyltrimethylammonium chloride (CTAC) as a surfactant and template, hydrochloric acid ( $\mathrm{HCl})$ as a catalyst, ethanol as a solvent, sodium hydroxide $(\mathrm{NaOH})$ as a gelation agent, and microwave power on the pore structure of as-prepared MSQ aerogels were investigated in detail. Microwave heating at low power results in the acceleration of sol-gel transition and achieves the gelation within a few minutes. Appropriate amounts of chemical reagents and microwave heating at high power allow the preparation of mesoporous MSQ aerogels with a BET-specific surface area of $681.6 \mathrm{~m}^{2} \cdot \mathrm{g}^{-1}$ and a mesopore size of $19 \mathrm{~nm}$, and the resultant MSQ aerogel still has a BET specific surface area as high as $134 \mathrm{~m}^{2} \cdot \mathrm{g}^{-1}$ after heat treatment at $600^{\circ} \mathrm{C}$ for $2 \mathrm{~h}$, showing high thermal stability. The MSQ aerogels / fibre composite possesses a low thermal conductivity of $0.039 \mathrm{~W} /(\mathrm{m} \cdot \mathrm{k})^{-1}$, displaying good thermal insulation. Microwave heating technology is a promising heating method for the preparation of other aerogels.
\end{abstract}

Keywords: MSQ aerogels; microwave drying; sol-gel

\section{Introduction}

Methylsilsesquioxane (MSQ) aerogel is a methyl hybrid silica aerogel prepared by sol-gel reaction containing methyl siloxane [1-4]. Compared with conventional silica aerogels, there are mutually exclusive methyl groups in MSQ aerogel framework that can improve its mechanical and intrinsic hydrophobic properties to some extent. MSQ aerogels have attracted widespread attention, especially in the fields of thermal insulation $[5,6]$, adsorbent [7-9] and architecture [10-12].

In recent years, many studies have been reported about the optimization of synthesis process, pore structure, and properties of MSQ aerogels. Rao [13] reported the preparation of flexible MSQ aerogels by using trifunctional methyltrimethoxysilane (MTMS) as a precursor, oxalic acid as a catalyst, methanol as a solvent and ammonia as a gelation agent via supercritical drying. Nadargi [14] prepared the MSQ aerogels by using MTES as a precursor with a two-stage acid-base catalyzed sol-gel process followed by supercritical drying. Zhong [15] successfully prepared flexible MSQ aerogels with polydimethylsiloxane (PDMS) and MTMS as co-precursors via atmospheric pressure drying. Kanamori [16] reported the preparation of organic-inorganic composite MSQ aerogels by a two-step acid-base reaction. Kazuyoshi [17] introduced the preparation of MSQ aerogels by using the MTMS as a precursor, with and a cationic surfactant used to promote the mixing of the MTMS-derived 
species and the solvent mixture. In our previous works $[18,19]$, flexible and elastic macroporous methylsilsesquioxane (MSQ) aerogels and translucent mesoporous MSQ aerogels with superhydrophobicity were successfully, prepared respectively. However, it should be noted that in most of the present reported MSQ aerogels, supercritical drying or ambient pressure drying was used to dry the wet gels. It is well known that supercritical drying needs special conditions such as high pressure and high temperature, which prevents aerogels from being used in industrial manufacturing and extended applications. Ambient pressure drying has become a popular research topic to replace supercritical drying for the preparation of aerogels, but low drying efficiency and poor structure retention limits its industrialization application. For these reasons, it is urgent to develop an effective heating method to achieve rapid drying of MSQ aerogels whilst retaining pore structure.

In the present work, we use microwave heating technology to accelerate the solgel transition and fast drying of wet gel to prepare mesoporous MSQ aerogels. In the preparation process, the effects of catalyst $(\mathrm{HCl})$, solvent (ethanol), surfactant $(\mathrm{CTAC})$ and microwave drying power on the pore structure of MSQ aerogels are investigated in detail. The controllable preparation of mesoporous MSQ aerogels can be rapidly achieved by controlling starting compositions and using microwave heating technology. The MSQ aerogels/fibre composite is further prepared by the same starting compositions and drying method.

\section{Materials and Methods}

\subsection{Materials}

Methyltrimethoxysilane (MTMS, Aladdin, Shanghai, China, 98\%) and hydrochloric acid ( $\mathrm{HCl}$, Aladdin, Shanghai, China) were used as a precursor and a catalyst, respectively. Hexadecyltrimethylammonium chloride (CTAC, Aladdin, Shanghai, China, 97\%) and sodium hydroxide (Aladdin, Shanghai, China, $97 \%$ ) acted as a surfactant and a gelation agent, respectively. Ethanol (Aladdin, Shanghai, China, $\geq 99.5 \%$ ), 2-propanol (Sinopharm Chemical Reagent Co., Ltd., Shanghai, China, $\geq 99.7 \%$ ) and n-heptane (Sinopharm Chemical Reagent Co., Ltd., Shanghai, China, $\geq 98.5 \%$ ) served as different kinds of solvent. All the chemical reagents were used as received.

\subsection{Preparation of MSQ Aerogel and Aerogel/Fiber Composites}

Preparation of MSQ aerogel: Table 1 shows the starting compositions and drying methods of all MSQ aerogel samples. A detailed preparation process was as follows. $0.24 \mathrm{~g}$ of CTAC, ethanol and $\mathrm{HCl}$ solution were uniformly mixed in a glass tube, and then $3.0 \mathrm{~mL}$ of MTMS was added with vigorous stirring in an ice-bath. After $30 \mathrm{~min}$, the transparent solution (MSQ sol) was formed, and then $\mathrm{NaOH}$ solution was added to the solution to increase the $\mathrm{pH}$ value of the system. After stirring for $1 \mathrm{~min}$, the as-prepared sol was gelated by microwave treatment at $350 \mathrm{~W}$ for $7 \mathrm{~min}$. After gelation, the wet gels were sealed and kept at $60^{\circ} \mathrm{C}$ for aging $24 \mathrm{~h}$. All of the wet gels were solvent-exchanged with 2-propanol and n-heptane to remove water and CTAC twice, respectively, and then were dried by microwave at $500-700 \mathrm{~W}$ for $60 \mathrm{~min}$ to obtain the MSQ aerogel.

Preparation of MSQ aerogel/fiber composites: $24 \mathrm{~g}$ of CTAC, ethanol, and $\mathrm{HCl}$ solution were mixed in a glass tube, and then $300 \mathrm{~mL}$ of MTMS was added with vigorous stirring for $30 \mathrm{~min}$ in an ice-bath to form the transparent sol. $\mathrm{NaOH}$ solution was added in the sol to increase the $\mathrm{pH}$ value for the gelation of MSQ sol. After stirring for 1-2 min, the quartz fibers were completely immersed in the resultant sol, and the sol immersed fiber was gelated by microwave treatment at $350 \mathrm{~W}$ for $15 \mathrm{~min}$. After gelation, the gel/fiber composite was sealed and kept at $60^{\circ} \mathrm{C}$ for $24 \mathrm{~h}$, and then was solvent-exchanged with 2-propanol and n-heptane to remove water and CTAC twice, respectively. Finally, the MSQ aerogel/fiber composite was prepared by microwave drying at $700 \mathrm{~W}$ for $75 \mathrm{~min}$. 
Table 1. Starting compositions and drying methods of MSQ aerogels.

\begin{tabular}{|c|c|c|c|c|c|c|c|}
\hline Sample & $\begin{array}{l}\text { VMTMS } \\
\text { /mL }\end{array}$ & $\begin{array}{l}\mathrm{VHCl} \\
/ \mathrm{mL}\end{array}$ & $\begin{array}{l}\text { VEtOH } \\
/ \mathrm{mL}\end{array}$ & $\begin{array}{l}\text { MCTAC } \\
/ g\end{array}$ & $\begin{array}{l}\mathrm{CNaOH} \\
/ \mathrm{mol} \cdot \mathrm{L}^{-1}\end{array}$ & $\begin{array}{l}\mathrm{VNaOH} \\
/ \mathrm{mL}\end{array}$ & $\begin{array}{l}\text { Microwave Drying } \\
\text { Power/W }\end{array}$ \\
\hline N1 & 3 & 1.5 & 1.5 & 1.6 & 0.5 & 0.5 & 700 \\
\hline N2 & 3 & 1.5 & 1.5 & 2.0 & 0.5 & 0.5 & 700 \\
\hline N3 & 3 & 1.5 & 1.5 & 2.4 & 0.5 & 0.5 & 700 \\
\hline N4 & 3 & 1.5 & 1.5 & 2.8 & 0.5 & 0.5 & 700 \\
\hline N5 & 3 & 1.5 & 1.5 & 3.2 & 0.5 & 0.5 & 700 \\
\hline N6 & 3 & 0.9 & 1.5 & 2.4 & 0.5 & 0.5 & 700 \\
\hline N7 & 3 & 1.2 & 1.5 & 2.4 & 0.5 & 0.5 & 700 \\
\hline N8 & 3 & 1.5 & 1.5 & 2.4 & 0.5 & 0.5 & 700 \\
\hline N9 & 3 & 1.8 & 1.5 & 2.4 & 0.5 & 0.5 & 700 \\
\hline N10 & 3 & 2.1 & 1.5 & 2.4 & 0.5 & 0.5 & 700 \\
\hline N11 & 3 & 1.5 & 1.0 & 2.4 & 0.5 & 0.5 & 700 \\
\hline N12 & 3 & 1.5 & 1.5 & 2.4 & 0.5 & 0.5 & 700 \\
\hline N13 & 3 & 1.5 & 2.0 & 2.4 & 0.5 & 0.5 & 700 \\
\hline N14 & 3 & 1.5 & 1.5 & 2.4 & 0.5 & 0.1 & 700 \\
\hline N15 & 3 & 1.5 & 1.5 & 2.4 & 0.5 & 0.2 & 700 \\
\hline N16 & 3 & 1.5 & 1.5 & 2.4 & 0.5 & 0.3 & 700 \\
\hline N17 & 3 & 1.5 & 1.5 & 2.4 & 0.5 & 0.4 & 700 \\
\hline N18 & 3 & 1.5 & 1.5 & 2.4 & 0.5 & 0.5 & 700 \\
\hline N19 & 3 & 1.5 & 1.5 & 2.4 & 1.0 & 0.1 & 700 \\
\hline N20 & 3 & 1.5 & 1.5 & 2.4 & 1.0 & 0.2 & 700 \\
\hline N21 & 3 & 1.5 & 1.5 & 2.4 & 1.0 & 0.3 & 700 \\
\hline $\mathrm{N} 22$ & 3 & 1.5 & 1.5 & 2.4 & 1.0 & 0.4 & 700 \\
\hline N23 & 3 & 1.5 & 1.5 & 2.4 & 1.0 & 0.5 & 700 \\
\hline N24 & 3 & 1.5 & 1.5 & 2.4 & 0.5 & 0.2 & 500 \\
\hline N25 & 3 & 1.5 & 1.5 & 2.4 & 0.5 & 0.2 & 700 \\
\hline N26 & 3 & 1.5 & 1.5 & 2.4 & 0.5 & 0.2 & Indoor evaporation \\
\hline N27 & 3 & 1.5 & 1.5 & 2.4 & 0.5 & 0.2 & $80^{\circ} \mathrm{C}$ oven drying \\
\hline
\end{tabular}

$\mathrm{V}_{\text {MTMS }}$ : volume of MTMS; $\mathrm{V}_{\mathrm{HCl}}$ : volume of $\mathrm{HCl}$; $\mathrm{V}_{\mathrm{EtOH}}$ : volume of ethanol; $\mathrm{M}_{\mathrm{CTAC}}$ : mass of $\mathrm{CTAC} ; \mathrm{V}_{\mathrm{NaOH}}$ : volume of $\mathrm{NaOH}$ solution; $\mathrm{C}_{\mathrm{NaOH}}$ : concentration of $\mathrm{NaOH}$ solution.

\subsection{Characterization}

The morphology of MSQ aerogels and composites was observed by a scanning electron microscope (SEM: Su8010, Hitachi, Tokyo, Japan). The Meso- and micropores of MSQ aerogels were characterized by an $\mathrm{N}_{2}$ adsorption-desorption apparatus (BET, ASAP2020HD88, Micromeritics Instruments Corporation, Norcross, GA, USA), and the samples were degassed at $200{ }^{\circ} \mathrm{C}$ under vacuum before adsorption-desorption measurement. The chemical compositions and thermal behaviour of MSQ aerogel were confirmed by Fourier-transform infrared spectroscopy (FT-IR, Nicolet 6700, ThermoFisher Scientific, Waltham, MA, USA), differential thermal analysis (DTA, Q200, TA, New Castle, DE, USA), and thermogravimetry (TG, TA-Q500, TA, New Castle, DE, USA). The thermal conductivity was obtained from a Hot Disk TPS $2500 \mathrm{~S}$.

\section{Results and Discussion}

\subsection{Preparation Mechanism of Mesoporous MSQ Aerogels by Microwave Drying}

Schematic illustration of rapid preparation of mesoporous MSQ aerogels by microwave drying was shown in Figure 1. In this acid-base system, CTAC is firstly dissolved in a mixture of ethanol and hydrochloric acid, and then the mixture is placed in ice bath until completely cooled. MTMS is added into the mixture and stirred for hydrolysis and polymerization. After that, $\mathrm{NaOH}$ solution is added to heighten the $\mathrm{pH}$ value of the system. In the meantime, the sol can be allowed to gelate after microwave treatment for a short time. After solvent replacement with 2-propanol and n-heptane and microwave drying, mesoporous MSQ aerogel can be obtained. 


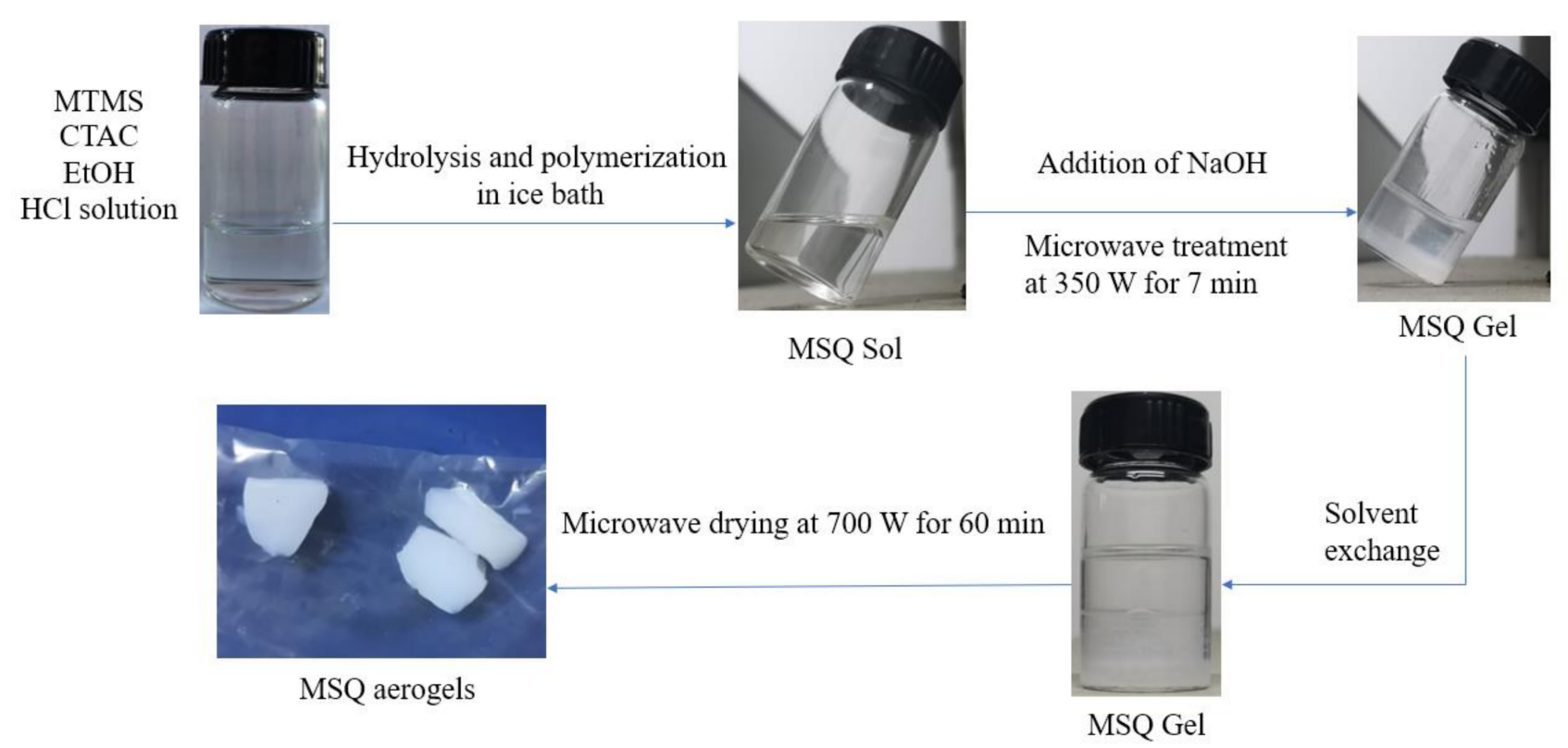

Figure 1. Schematic illustration of preparation process of methylsilsesquioxane (MSQ) aerogels by microwave drying.

Microwave heating technology is a kind of heating method which uses electromagnetic wave as a heating source and the dried material itself as a heat generator. Compared with traditional heating methods through thermal radiation, microwave heating technology uniformly produces the heat by dielectric loss in electromagnetic field. Therefore, in this sol-gel process, the microwave heating technology can be used to not only accelerate the sol-gel transition but also achieve the fast drying of aerogels, which shortens the preparation procedure for aerogels. Microwave heating technology should be considered as an efficient gelation and drying technology for aerogels.

\subsection{Optimization of Synthesis Process of MSQAerogels}

\subsubsection{Effect of CTAC on Pore Structure of MSQ Aerogels}

Surfactant is known to have a beneficial effect on the formation of network frame, pore structure and particle size of aerogels. In this system, the CTAC is used as the surfactant for preparation of MSQ aerogels, and the effect of CTAC content on the pore structure of MQS aerogels is investigated. Figure 2 shows the pore structure of MSQ aerogels prepared via sol-gel process with different CTAC contents ( $\left.\mathrm{M}_{\mathrm{CTAC}}\right)$. We observed that all the MSQ aerogels possess a mesopore structure with uniform three-dimensional networks. With the increase in CTAC content, the size of mesopores increases, and the typical mesopore structure constructed by uniform gel network and homogeneous nanoparticles can be obtained when the CTAC content reaches $2.4 \mathrm{~g}$. When the amount of CTAC further increases to 2.8 or $3.2 \mathrm{~g}$, the mesopores of MSQ aerogels become smaller with some granular aggregates, indicating the deterioration of pore structure. The excessive involvement of CTAC in network construction resulted in a reduction in the liquid phase and accelerated the agglomeration of nanoparticles. As a result, it is concluded that $2.4 \mathrm{~g}$ of CTAC is an appropriate additive content of surfactant for the rapid preparation of MSQ aerogels.

\subsubsection{Effect of Ethanol on Pore Structure of MSQ Aerogels}

In previous works [15-19], methanol was often used as the solvent in the preparation of MSQ aerogels. However, methanol is a volatile chemical with certain toxicity, which will cause environmental pollution. Compared with methanol, ethanol has similar physical and chemical properties, and it is indeed less toxic. In this work, ethanol is used as the solvent for preparation of mesoporous MSQ aerogels, and the effect of ethanol volume on the pore structure of MSQ aerogel is analyzed. Figure 3 shows the pore structure of MSQ 
aerogels with different volumes of ethanol $\left(\mathrm{V}_{\mathrm{EtOH}}\right)$. It can be seen from the figures that as the content of ethanol increases gradually, the pore structure of MSQ aerogels has no obvious change, proving that the volume of ethanol has little relationship with the pore structure of MSQ aerogels. As a solvent, more ethanol will produce the tendency of phase separation, thus affecting the retention of pore structure in aerogels during drying. A small change in ethanol content does not affect the pore structure of MSQ aerogels obviously. In contrast, the MSQ aerogels with $1.5 \mathrm{~mL}$ ethanol show more uniform mesopore structure. This seems to conclude that $1.5 \mathrm{~mL}$ of ethanol is a suitable volume of solvent for rapid preparation of MSQ aerogels.

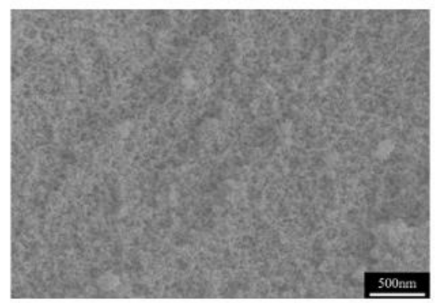

(a) $M \mathrm{CTAC}=1.6 \mathrm{~g}$

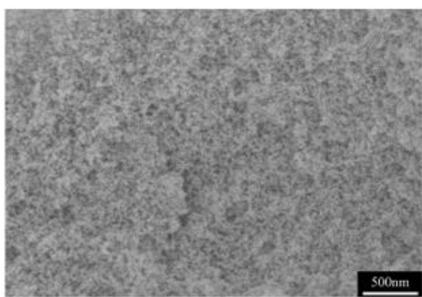

(b) $M c$ TAC $=2.0 \mathrm{~g}$

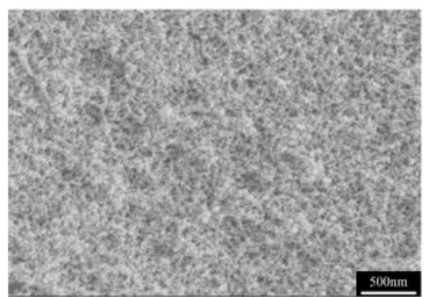

(c) $M c T_{A C}=2.4 \mathrm{~g}$

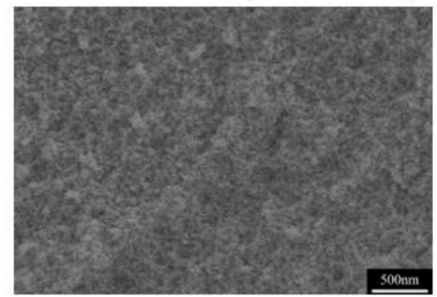

(d) $M$ CTAC $=2.8 \mathrm{~g}$

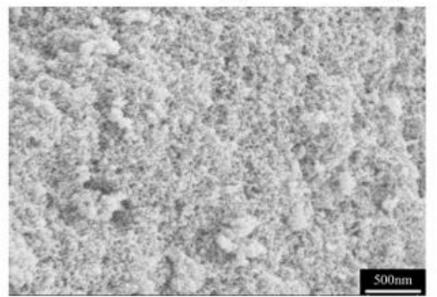

(e) $M c t A C=3.2 \mathrm{~g}$

Figure 2. SEM images of MSQ aerogels prepared by $700 \mathrm{~W}$ microwave drying via sol-gel process with different CTAC contents. (a) $1.6 \mathrm{~g}$, (b) $2.0 \mathrm{~g}$, (c) $2.4 \mathrm{~g}$, (d) $2.8 \mathrm{~g}$, (e) $3.2 \mathrm{~g}$.

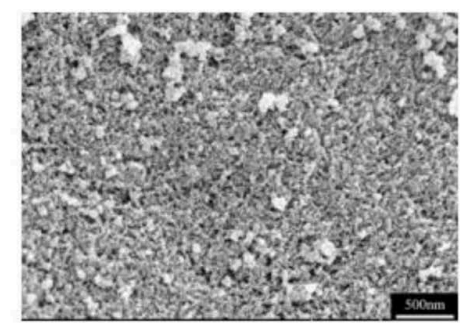

(a) $V_{\mathrm{EtOH}}=1.0 \mathrm{~mL}$

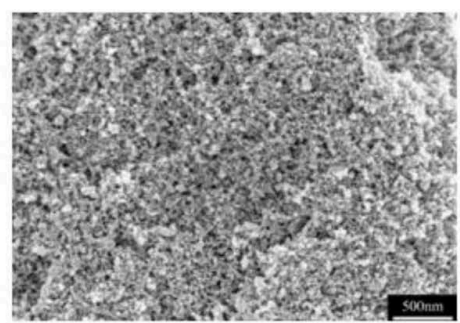

(b) $V_{\mathrm{EtOH}}=1.5 \mathrm{~mL}$

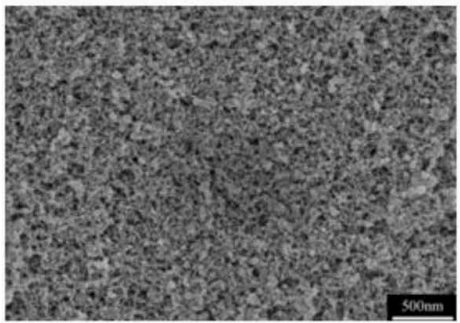

(c) $V_{\mathrm{EtOH}}=2.0 \mathrm{~mL}$

Figure 3. SEM images of MSQ aerogels prepared by $700 \mathrm{~W}$ microwave drying via sol-gel process with different volumes of ethanol: (a) $1.0 \mathrm{~mL}$, (b) $1.5 \mathrm{~mL}$, (c) $2.0 \mathrm{~mL}$.

\subsubsection{Effect of Catalyst $(\mathrm{HCl})$ on Pore Structure of MSQ Aerogels}

In sol-gel reaction, the hydrochloric acid $(\mathrm{HCl})$ is widely used as a catalyst for the hydrolysis of precursors. In this system, owing to the high exothermic reaction of MTMS hydrolysis, the reaction condition of an ice bath is essential to control the hydrolysis and polymerization, while at the same time, the hydrolysis of MTMS needs to be catalyzed by $\mathrm{HCl}$. Here, the effect of additive volume of $\mathrm{HCl}$ on the pore structure of MSQ aerogels is inspected. Figure 4 shows the pore structure of MSQ aerogels with different volumes of $\mathrm{HCl}$ solution $\left(V_{\mathrm{HCl}}\right)$. It is noted that with the increase in volume of $\mathrm{HCl}$, the pore structure of MSQ aerogel does not change significantly. This may be due to the whole reaction being in the ice bath environment, the velocity of hydrolysis reaction is controlled very slowly. By comparison, the MSQ aerogels with $1.5 \mathrm{~mL} \mathrm{HCl}$ solution show a more uniform mesopore 
structure. It can be concluded that $1.5 \mathrm{~mL}$ of $\mathrm{HCl}$ solution is an appropriate volume of catalyst for rapid preparation of MSQ aerogels.

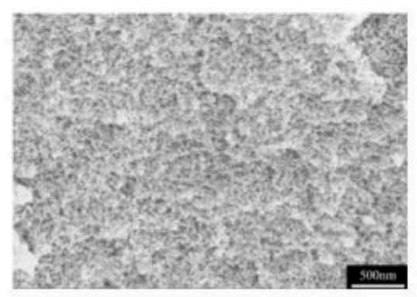

(a) $V_{\mathrm{HCl}}=0.9 \mathrm{~mL}$

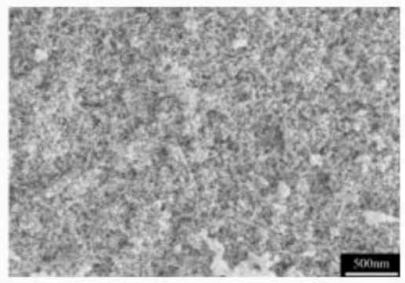

(b) $V_{\mathrm{HCl}}=1.2 \mathrm{~mL}$

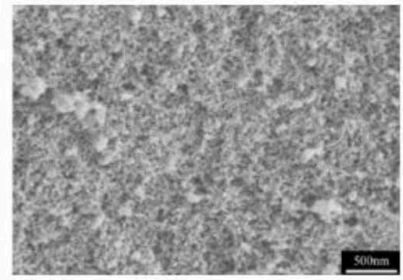

(c) $V_{\mathrm{HCl}}=1.5 \mathrm{~mL}$

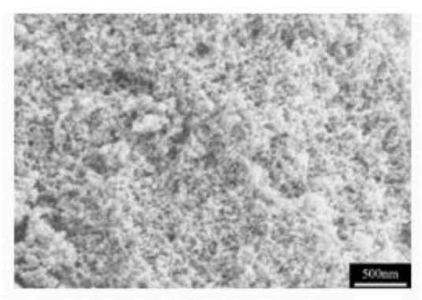

(d) $V_{\mathrm{HCl}}=1.8 \mathrm{~mL}$

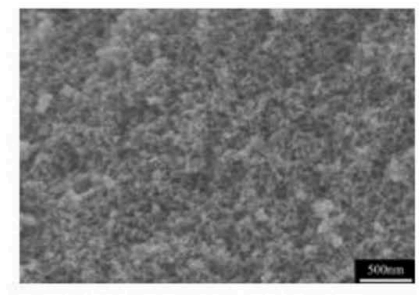

(e) $V_{\mathrm{HCl}}=2.1 \mathrm{~mL}$

Figure 4. SEM images of MSQ aerogels prepared by $700 \mathrm{~W}$ microwave drying via sol-gel process with different volumes of $\mathrm{HCl}$ solution. (a) $0.9 \mathrm{~mL}$, (b) $1.2 \mathrm{~mL}$, (c) $1.5 \mathrm{~mL}$, (d) $1.8 \mathrm{~mL}$, (e) $2.1 \mathrm{~mL}$.

\subsubsection{Effect of Sodium Hydroxide (NaOH) on Pore Structure of MSQ Aerogels}

In our previous works $[18,19]$, the propylene oxide $(\mathrm{PO})$ was often used as the gelation agent in the preparation of MSQ aerogels. However, $\mathrm{PO}$ is also a volatile chemical with certain toxicity, which will cause environmental pollution. In this system, the frequently used $\mathrm{NaOH}$ solution is used as the gelation agent to accelerate the sol-gel transition, the effects of the volume and concentration of $\mathrm{NaOH}$ solution on the pore structure of MSQ aerogels are surveyed. Figures 5 and 6 show the pore structure of MSQ aerogels with different volumes of 0.5 and $1.0 \mathrm{~mol} \cdot \mathrm{L}^{-1} \mathrm{NaOH}$ solutions $\left(\mathrm{V}_{\mathrm{NaOH}}\right)$, respectively. For 0.5 or $1.0 \mathrm{~mol} \cdot \mathrm{L}^{-1} \mathrm{NaOH}$ solution, with the increase in the volume of $\mathrm{NaOH}$ solution, the pore structure of both MSQ aerogels becomes more compact. In particular, when the volume of $0.5 \mathrm{~mol} \cdot \mathrm{L}^{-1} \mathrm{NaOH}$ solution reaches $0.4 \mathrm{~mL}\left(0.3 \mathrm{~mL}\right.$ for $1.0 \mathrm{~mol} \cdot \mathrm{L}^{-1} \mathrm{NaOH}$ solution), the pore structure of MSQ aerogel is extremely dense. In contrast, MSQ aerogel has uniform pore structure with $0.2 \mathrm{~mL}$ of $0.5 \mathrm{~mol} \cdot \mathrm{L}^{-1} \mathrm{NaOH}$ solution $\left(0.1 \mathrm{~mL}\right.$ of $1.0 \mathrm{~mol} \cdot \mathrm{L}^{-1}$ $\mathrm{NaOH}$ solution), indicating better volume and concentration of gelation agent for rapid preparation of MSQ aerogels.

\subsubsection{Effects of Microwave Power and Drying Method on Pore Structure of MSQ Aerogels}

Drying technology is one of the most important processes in aerogel preparation and determines the pore structure and production cost of aerogels. In this work, microwave heating technology is used to rapidly dry the wet gels, and the effect of microwave power on the pore structure of MSQ aerogels is researched. Additionally, the microwave drying is also compared with the indoor evaporation drying and oven drying. Figure 7 shows the pore structure of MSQ aerogels prepared by microwave at different powers, indoor evaporation drying and $80^{\circ} \mathrm{C}$ oven drying, respectively. As can be seen from the figures, the MSQ aerogels prepared by microwave drying have more uniform pore structure than those dried by indoor evaporation drying or oven drying. Meanwhile, the power of microwave has little influence on the mesopores of MSQ aerogels. In contrast, the MSQ microwave dried at $500 \mathrm{~W}$ has more well-distributed pore structure than that at $700 \mathrm{~W}$. It is certain that $500 \mathrm{~W}$ of microwave power is a suitable power of microwave drying for rapid preparation of MSQ aerogels. 


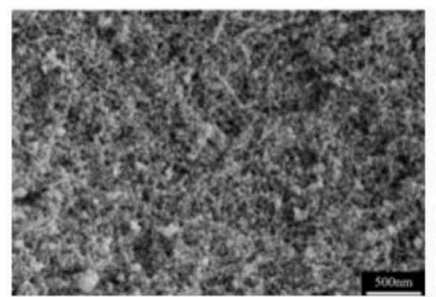

(a) $V_{\mathrm{NaOH}}=0.1 \mathrm{~mL}$

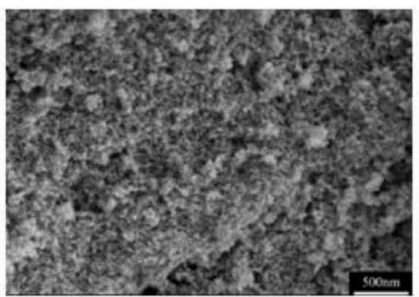

(b) $V_{\mathrm{NaOH}}=0.2 \mathrm{~mL}$

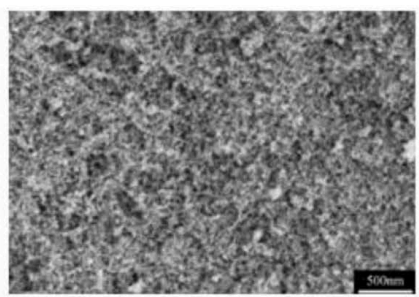

(c) $V_{\mathrm{NaOH}}=0.3 \mathrm{~mL}$

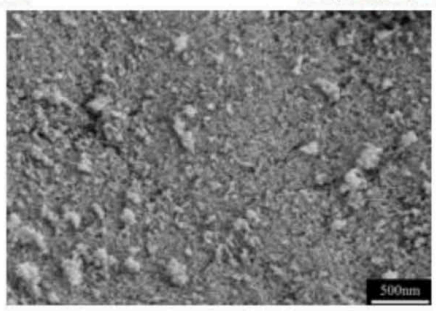

(d) $V_{\mathrm{NaOH}}=0.4 \mathrm{~mL}$

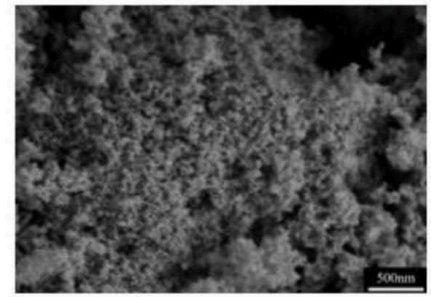

(e) $V_{\mathrm{NaOH}}=0.5 \mathrm{~mL}$

Figure 5. SEM images of MSQ aerogels prepared by $700 \mathrm{~W}$ microwave drying via sol-gel process with different volumes of $0.5 \mathrm{~mol} \cdot \mathrm{L}^{-1} \mathrm{NaOH}$ solution. (a) $0.1 \mathrm{~mL}$, (b) $0.2 \mathrm{~mL}$, (c) $0.3 \mathrm{~mL}$, (d) $0.4 \mathrm{~mL}$, (e) $0.5 \mathrm{~mL}$.

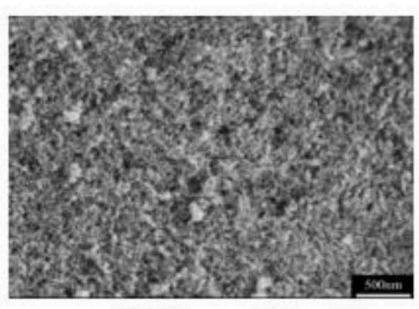

(f) $V_{\mathrm{NaOH}}=0.1 \mathrm{~mL}$

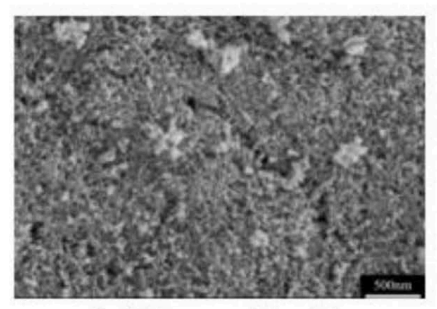

(g) $V_{\mathrm{NaOH}}=0.2 \mathrm{~mL}$

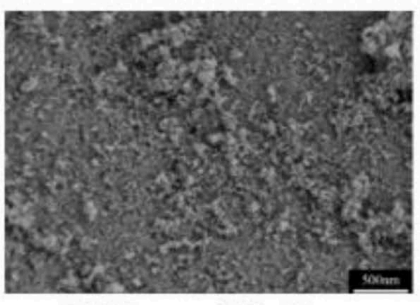

(h) $\mathrm{V}_{\mathrm{NaOH}}=0.3 \mathrm{~mL}$

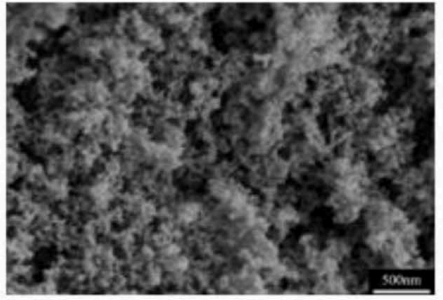

(i) $V_{\mathrm{NaOH}}=0.4 \mathrm{~mL}$

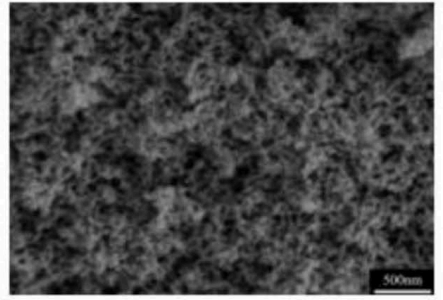

(j) $V_{\mathrm{NaOH}}=0.5 \mathrm{~mL}$

Figure 6. SEM images of MSQ aerogels prepared by $700 \mathrm{~W}$ microwave drying via sol-gel process with different volumes of $1.0 \mathrm{~mol} \cdot \mathrm{L}^{-1} \mathrm{NaOH}$ solution. (f) $0.1 \mathrm{~mL}$, (g) $0.2 \mathrm{~mL}$, (h) $0.3 \mathrm{~mL}$, (i) $0.4 \mathrm{~mL}$, (j) $0.5 \mathrm{~mL}$.

Figure 8 shows the $\mathrm{N}_{2}$ adsorption-desorption isotherms (a) and BJH mesopore size distributions (b) of different MSQ aerogels prepared by microwave at different powers, indoor evaporation drying and oven drying. It is clear that the MSQ aerogel prepared by microwave drying at $500 \mathrm{~W}$ presents a $\mathrm{H}_{2}$ adsorption-desorption hysteresis loop according to the IUPAC classification, confirming the existence of uniform and orderly mesopores. The calculated BET specific surface area and a mesopore size of MSQ aerogel by microwave drying at $500 \mathrm{~W}$ are $668 \mathrm{~m}^{2} \cdot \mathrm{g}^{-1}$ and $19 \mathrm{~nm}$, respectively. The BET specific surface area and a mesopore size of MSQ aerogel by microwave drying at $700 \mathrm{~W}$ are $557 \mathrm{~m}^{2} \cdot \mathrm{g}^{-1}$ and $17 \mathrm{~nm}$, respectively, which indicates that the micropores or mesopores decrease and the pore structure collapses at high microwave power. By comparison, the two MSQ aerogels prepared by indoor evaporation drying and oven drying show a dense microstructure and lower specific surface area. This confirms that microwave heating technology is an effective drying method for the rapid preparation of MSQ aerogels. 


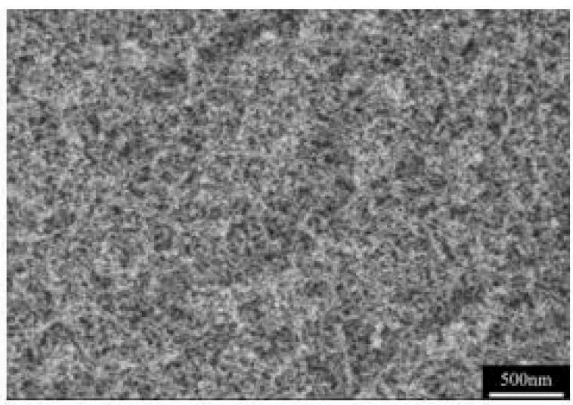

(a) $500 \mathrm{~W}$ microwave drying

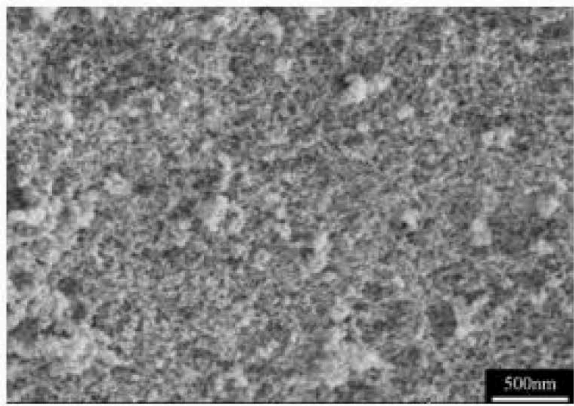

(c) indoor evaporation drying

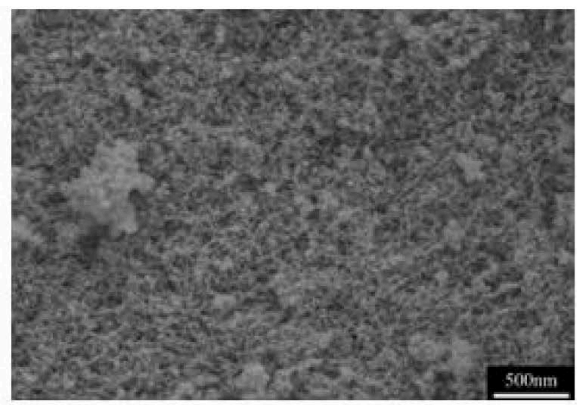

(b) $700 \mathrm{~W}$ microwave drying

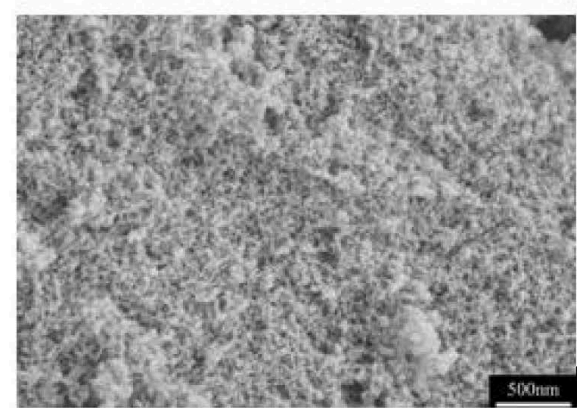

(d) $80^{\circ} \mathrm{C}$ oven drying

Figure 7. SEM images of MSQ aerogels prepared by microwave at different powers, indoor evaporation drying and oven drying. (a) $500 \mathrm{~W}$ microwave drying, (b) $700 \mathrm{~W}$ microwave drying, (c) indoor evaporation drying, (d) $80^{\circ} \mathrm{C}$ oven drying.

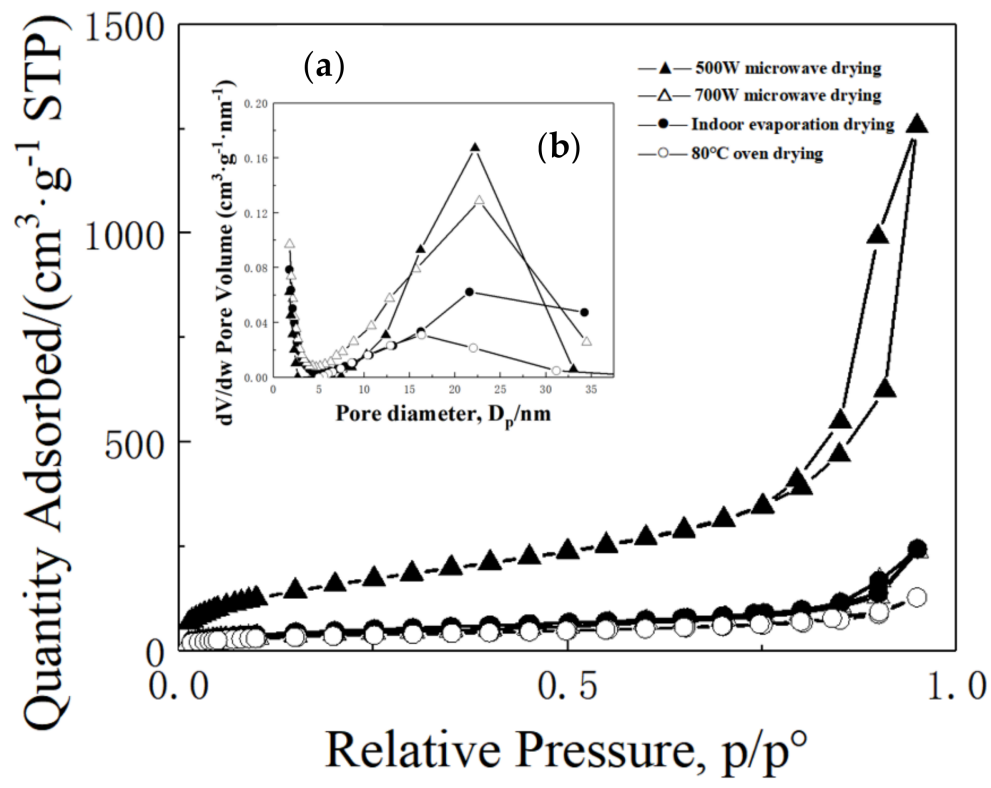

Figure 8. $\mathrm{N}_{2}$ adsorption-desorption isotherms (a) and BJH mesopore size distributions (b) of MSQ aerogels prepared by microwave at different powers, indoor evaporation drying and oven drying.

\subsection{Thermal Stability and Pore Structures of Typical MSQ Aerogels}

Figure 9 shows differential thermal analysis (DTA) and thermogravimetry (TG) curves of a typical MSQ aerogel. It is noted that when the temperature rises from 150 to $230^{\circ} \mathrm{C}$, there appears about $8 \%$ mass loss, which can be attributed to the evaporation of residual water. When the temperature further increases to $350^{\circ} \mathrm{C}$, the methyl groups of MSQ aerogel 
start to be oxygenated, which directly results in a particularly sharp peak in DTA curve and a nearly $80 \%$ mass loss. From infrared (IR) spectrum of the MSQ aerogels, the absorption peaks around 3439 and $1637 \mathrm{~cm}^{-1}$ are assigned as the stretching vibration and bending vibration of the O-H group of water, respectively. The absorption peaks around 2927 and $2972 \mathrm{~cm}^{-1}$ are attributed to the stretching vibration of the C-H group, and the absorption peaks around 1273 and $1137 \mathrm{~cm}^{-1}$ are due to the bending vibration of the $\mathrm{C}-\mathrm{H}$ group, indicating the existence of a methyl group. The absorption peaks around 1137, 1037 and $444 \mathrm{~cm}^{-1}$ are ascribed to the stretching vibration of the O-Si-O group. The results confirm the successful preparation of pure MSQ aerogel.

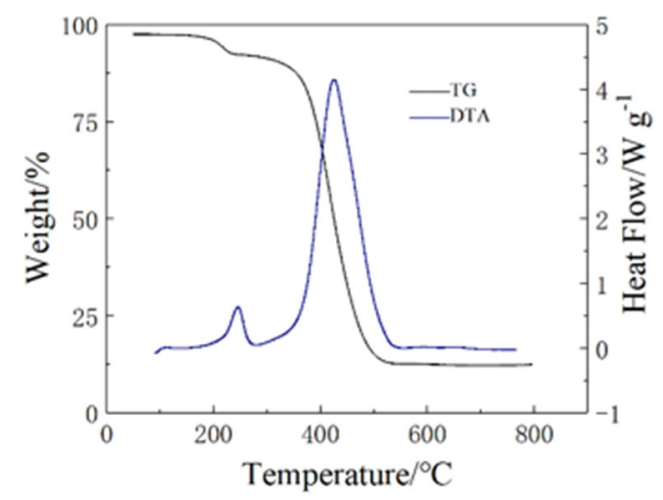

(a) DTA/TG curves

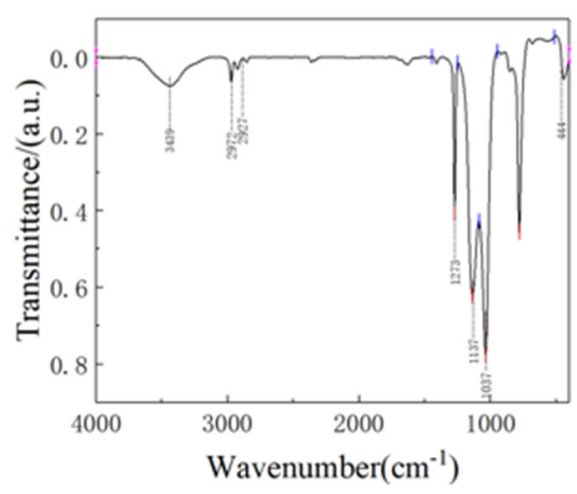

(b) IR spectra curve

Figure 9. Differential thermal analysis (DTA)/thermogravimetry (TG) curves (a) and infrared (IR) specta curve (b) of a typical MSQ aerogel.

The thermal stability of MSQ aerogel was investigated by heat-treating MSQ aerogel at different temperatures, and the pore structure change in heat-treated MSQ aerogels at $300-600{ }^{\circ} \mathrm{C}$ has been further studied. Figure 10 shows SEM images of typical MSQ aerogels after heat-treatment at different temperatures. It is seen that, with the increase in temperature, the pore structure of MSQ aerogels gradually shrinks and compacts. When the temperature reached 300 or $400{ }^{\circ} \mathrm{C}$, the pore structure is not appreciably changed, but becomes very compact at $600{ }^{\circ} \mathrm{C}$, which is the result of the exothermic reaction of methyl decomposition.

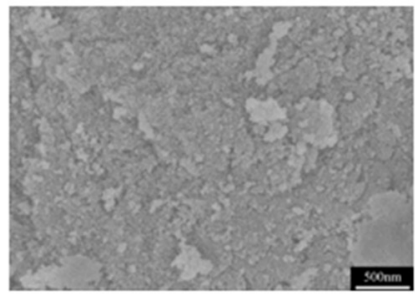

(a) $300^{\circ} \mathrm{C}$

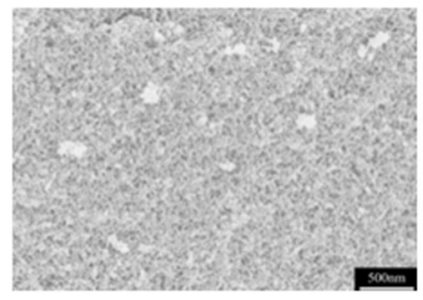

(b) $400^{\circ} \mathrm{C}$

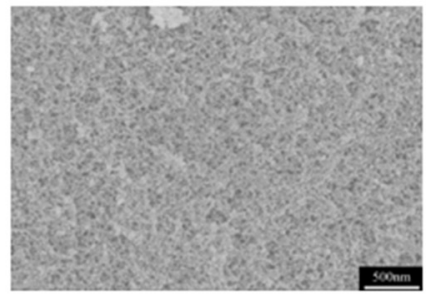

(c) $600^{\circ} \mathrm{C}$

Figure 10. SEM images of MSQ aerogels prepared by $500 \mathrm{~W}$ microwave drying after heat-treated at different temperatures. (a) $300{ }^{\circ} \mathrm{C}$, (b) $400{ }^{\circ} \mathrm{C}$, (c) $600{ }^{\circ} \mathrm{C}$.

Figure 11 shows the $\mathrm{N}_{2}$ adsorption-desorption isotherms (a) and BJH mesopore size distributions (b) of typical MSQ aerogels heat-treated at varied temperatures. It is noted that the dried MSQ aerogel without heat-treatment presents a H2 adsorption-desorption hysteresis loop according to the IUPAC classification, confirming the existence of uniform and orderly mesopores. The calculated BET specific surface area and a mesopore size are $682 \mathrm{~m}^{2} \cdot \mathrm{g}^{-1}$ and $19 \mathrm{~nm}$, respectively. After heat-treatment at 300 and $400{ }^{\circ} \mathrm{C}$, the isotherms and mesopore size distribution of MSQ aerogel have no obvious difference, while the overall shape of the adsorption-desorption hysteresis loop turns from $\mathrm{H} 2$ to $\mathrm{H} 3$, indicating 
the shrinking of mesopores and collapse of the pore structure after heat-treatment at $600{ }^{\circ} \mathrm{C}$. The resultant MSQ aerogel after heat treatment at $600^{\circ} \mathrm{C}$ for $2 \mathrm{~h}$ still has a specific surface area as high as $134 \mathrm{~m}^{2} \cdot \mathrm{g}^{-1}$, showing high thermal stability.

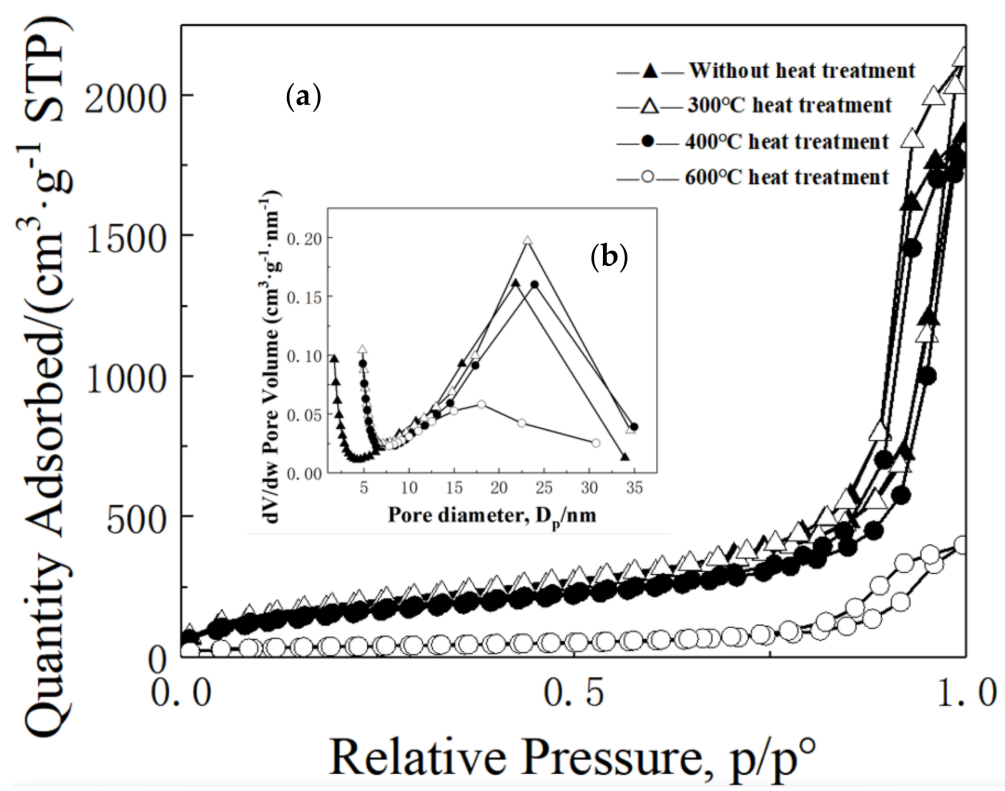

Figure 11. $\mathrm{N}_{2}$ adsorption-desorption isotherms (a) and BJH mesopore size distributions (b) of MSQ aerogels after heat treatment at different temperatures.

\subsection{Thermal Insulation Performance of MSQ Aerogel/Fiber Composites}

Figure 12 shows the SEM image of MSQ aerogel/fiber composite. It is seen that some of the MSQ aerogels are attached on the surface of the fibers, and most of them fill in the network of the fibers or suspend on the fiber in the form of monoliths. Compared with the pure MSQ aerogel, the pore structure of MSQ aerogels in the composite become relatively denser, which possibly results from a collapse of mesopores or micropores during the microwave drying or a incomplete solvent exchange. The measured thermal conductivity of MSQ aerogel/fiber composite is $0.039 \mathrm{~W} /(\mathrm{m} \cdot \mathrm{K})^{-1}$, showing a high thermal insulation performance.
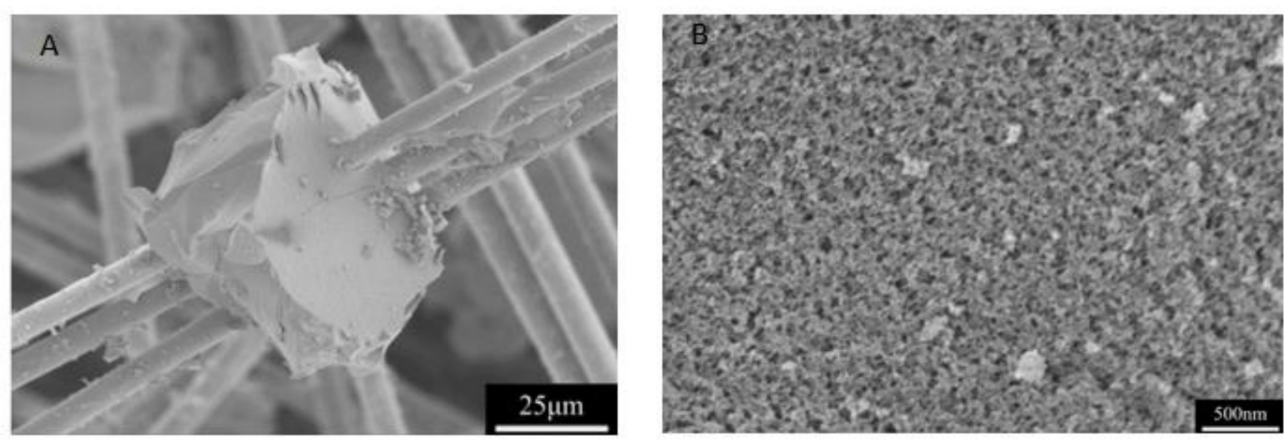

Figure 12. SEM images of the MSQ aerogel/fiber composites prepared by $700 \mathrm{~W}$ microwave drying. (A) combination of aerogel with fibre, (B) microstructure of aerogel in the composite.

\section{Conclusions}

Microwave heating technology was used to accelerate the sol-gel transition and fast dry the wet gel to rapidly prepare methylsilsesquioxane (MSQ) aerogels with homogeneous mesoporous pores. In order to obtain a controllable and optimal formulation of MSQ aerogels, the influences of surfactant, catalyst, gelation agent, solvent, and microwave 
power on pore structure were thoroughly and carefully investigated. An appropriate proportion of MTMS: $\mathrm{HCl}: \mathrm{H}_{2} \mathrm{O}: \mathrm{EtOH}: \mathrm{CTAC}: \mathrm{NaOH}$ and microwave power of $500 \mathrm{~W}$ allow the preparation of MSQ aerogel with a specific surface area of $682 \mathrm{~m}^{2} \cdot \mathrm{g}^{-1}$ and a mesopore size of $19 \mathrm{~nm}$. The resultant MSQ aerogel displays high thermal stability, and a specific surface area still reaches $134 \mathrm{~m}^{2} \cdot \mathrm{g}^{-1}$ after heat treatment at $600^{\circ} \mathrm{C}$. The MSQ aerogels/fibre composite has good thermal insulation with a thermal conductivity of $0.039 \mathrm{~W} /(\mathrm{m} \cdot \mathrm{k})^{-1}$. The microwave heating technology is promising for wider use in quick preparation of aerogels.

Author Contributions: X.G. and Z.L. conceived and designed the experiments; Z.L. and W.L. performed the experiments; X.G., Z.L. and W.L. analyzed the data; R.D. and Y.Z. contributed reagents/materials/analysis tools; X.G. and Z.L. wrote the paper; H.Y. supervised the experiment. All authors have read and agreed to the published version of the manuscript.

Funding: This research was funded by National Natural Science Foundation of China (21875217 and 51372225), High Science \& Technique Brainstorm Project of Zhejiang Province of China (2017C01002), and Industry-University-Research Collaboration Project of Jiangsu Province (FZ20180405).

Data Availability Statement: The data presented in this study are available on request from the corresponding author.

Conflicts of Interest: The authors declare no conflict of interest.

Sample Availability: Samples of the compounds are not available from the authors.

\section{References}

1. Hrubesh, L.W. Aerogel applications. J. Non Cryst. Solids 1998, 225, 335-342. [CrossRef]

2. Pierre, A.C.; Pajonk, G.M. Chemistry of Aerogels and Their Applications. Chem. Rev. 2002, 102, 4243-4266. [CrossRef] [PubMed]

3. Gurav, J.L.; Jung, I.-K.; Park, H.-H.; Kang, E.S.; Nadargi, D.Y. Silica Aerogel: Synthesis and Applications. J. Nanomater. 2010, 2010, 1-11. [CrossRef]

4. Dorcheh, A.S.; Abbasi, M. Silica aerogel; synthesis, properties and characterization. J. Mater. Process. Technol. 2008, 199, 10-26. [CrossRef]

5. He, Y.-L.; Xie, T. Advances of thermal conductivity models of nanoscale silica aerogel insulation material. Appl. Therm. Eng. 2015, 81, 28-50. [CrossRef]

6. Wu, S.H.; Mou, C.Y.; Lin, H.P. Synthesis of mesoporous silica nanoparticles. Chem. Soc. Rev. 2013, 42, 3862-3875. [CrossRef] [PubMed]

7. Li, C.; Wei, M.; Evans, D.G.; Duan, X. Layered double hydroxide-based nanomaterials as highly efficient catalysts and adsorbents. Small 2014, 10, 4469-4486. [CrossRef]

8. Zhang, C.; Dai, C.; Zhang, H.; Peng, S.; Wei, X.; Hu, Y. Regeneration of mesoporous silica aerogel for hydrocarbon adsorption and recovery. Mar. Pollut. Bull. 2017, 122, 129-138. [CrossRef] [PubMed]

9. Chen, M.; Li, Z.; Li, J.; Li, J.; Li, Q.; Zhang, L. The extraction of uranium using graphene aerogel loading organic solution. Talanta 2017, 166, 284-291. [CrossRef]

10. Asdrubali, F.; D'Alessandro, F.; Schiavoni, S. A review of unconventional sustainable building insulation materials. Sustain. Mater. Technol. 2015, 4, 1-17. [CrossRef]

11. Baetens, R.; Jelle, B.P.; Gustavsen, A. Aerogel insulation for building applications: A state-of-the-art review. Energy Build. 2011, 43, 761-769. [CrossRef]

12. Cuce, E.; Cuce, P.M.; Wood, C.J.; Riffat, S.B. Toward aerogel based thermal superinsulation in buildings: A comprehensive review. Renew. Sustain. Energy Rev. 2014, 34, 273-299. [CrossRef]

13. Rao, A.V.; Kulkarni, M.M.; Amalnerkar, D.; Seth, T. Superhydrophobic silica aerogels based on methyltrimethoxysilane precursor. J. Non Cryst. Solids 2003, 330, 187-195. [CrossRef]

14. Nadargi, D.Y.; Rao, A.V. Methyltriethoxysilane: New precursor for synthesizing silica aerogels. J. Alloys Compd. 2009, 467, 397-404. [CrossRef]

15. Zhong, L.; Chen, X.; Song, H.; Guo, K.; Hu, Z. Highly flexible silica aerogels derived from methyltriethoxysilane and polydimethylsiloxane. New J. Chem. 2015, 39, 7832-7838. [CrossRef] 
16. Kanamori, K.; Hayase, G.; Nakanishi, K.; Hanada, T. Pore Structure and Mechanical Properties of Poly(methylsilsesquioxane) Aerogels. IOP Conf. Ser. Mater. Sci. Eng. 2011, 18, 032001. [CrossRef]

17. Kanamori, K.; Aizawa, M.; Nakanishi, K.; Hanada, T. New Transparent Methylsilsesquioxane Aerogels and Xerogels with Improved Mechanical Properties. Adv. Mater. 2007, 19, 1589-1593. [CrossRef]

18. Guo, X.; Shan, J.; Lai, Z.; Lei, W.; Ding, R.; Zhang, Y.; Yang, H. Facile Synthesis of Flexible Methylsilsesquioxane Aerogels with Surface Modifications for Sound- Absorbance, Fast Dye Adsorption and Oil/Water Separation. Molecules 2018, 23, 945. [CrossRef] [PubMed]

19. Guo, X.; Shan, J.; Lei, W.; Ding, R.; Zhang, Y.; Yang, H. Facile Synthesis of Methylsilsesquioxane Aerogels with Uniform Mesopores by Microwave Drying. Polymers 2019, 11, 375. [CrossRef] [PubMed] 